\title{
COVID-19: Evolution and Prevention
}

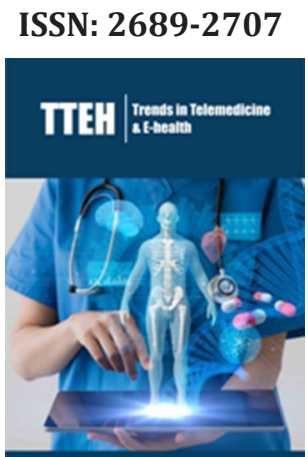

*Corresponding author: Sunil J Wimalawansa, Professor of Medicine, Endocrinology \& Nutrition, Cardiometabolic and Endocrine Institute, USA

Submission: 悳 July 29, 2020

Published: 䟱August 12, 2020

Volume 2 - Issue 3

How to cite this article: Wimalawansa SJ. COVID-19: Evolution and Prevention. Trends Telemed E-Health 2(3). TTEH. 000539. 2020. DOI: 10.31031/TTEH.2020.02.000539

Copyright@ Sunil J Wimalawansa, This article is distributed under the terms of the Creative Commons Attribution 4.0 International License, which permits unrestricted use and redistribution provided that the original author and source are credited.

\author{
Wimalawansa SJ* \\ Professor of Medicine, Endocrinology \& Nutrition, USA
}

\begin{abstract}
Advanced age, having comorbidities, and vitamin D deficiency are three most important reasons for increased vulnerability to COVID-19 and also worsen complications and increase the risk of death. Despite the vast amount of information available and lessons learned, many countries are still not fully utilizing these to manage secondary peaks of COVID-19 infection. Factors associated with worse COVID-19 prognosis include, older age, ethnicity, male sex, having comorbidities, obesity, diabetes, hypertension, and smoking; all these are associate with vitamin D deficiency. COVID-19 symptomatology varies from mostly asymptomatic, to, up to $2 \%$ fatality. The latter is characterized by cytokine storm, an immune reaction, diffuse arterial thromboembolism, acute respiratory distress syndrome, pulmonary oedema, and death. Vitamin D deficiency positively corelates with disease severity, complications, and deaths. Considering the wider beneficial effects from maintaining vitamin D adequacy, prophylactic broader supplementation programs should be considered by all administrations on public health point of view. With excellent tolerability and safety, even with high doses of vitamin D, and a highly economical nutrient supplement, this approach should be prioritized.
\end{abstract}

Keywords: Cardiovascular; Coronavirus; Endocrine; Infection; Inflammation; Innate immunity; Vitamin D; Pandemic; Prevention; Sars-Cov-2; Vaccine

\section{Introduction}

The coronavirus outbreak from COVID-19 that started in Wuhan city in Hubei Province, in China have spread across the world. Coronaviruses are zoonotic viruses, circulating within wild animals mostly in China and in the middle eastern region. However, the virus might have either naturally or synthetically mutated its spike-protein, acquiring the capacity to anchor on to membrane-bound ACE2 receptors in the presence of heparin sulphate proteoglycan. This allows COVID-19 to penetrate humans cell membranes or internalize with ACE-2 receptors inserting its genetic material within human epithelial cells [1,2]. Using a similar mechanism virus then transfect from human to human. In addition, the soluble form of ACE2 circulate in the blood $[3,4]$, which is capable of neutralize coronaviruses by binding to them and chaperoning the complex to macrophages to destroy. This action removes viruses from the circulation and prevents the entry of COVID-19 into healthy cells [5,6]. There is a possibility that in due course, its genome could mutate again, changing its virulence, antibody generation, and the pattern of spread. Based on the rapidity and the infectivity, COVID-19 has becomea serious public health threat and socioeconomic concern that affecting the global economy.

Precautions taken to prevent the spread of the common cold and flu-like illnesses, including minimizing exposure by limiting time in public places and public transportation and frequently washing hands, wearing face masks inn public, are the essential basic steps people should adhere to prevent the spread and acquiring this disease. It is noteworthy that, many face masks currently sold, unless designed to filter smaller particles such as viruses, while could curtail the spread of larger droplets, thus, partially protective, unlikely to prevent the inhalation of coronaviruses and transmitting those to others.

\section{What is a Coronavirus-(2019-nCoV/COVID-19)?}

Coronaviruses have been in existence for long time. A variety of coronaviruses have been identified, especially those causing common colds and other respiratory infections. Most of the known coronaviruses are mostly abundant among wild and exotic animals in certain countries. The main reservoir in nature for the $2019-\mathrm{nCoV}$ is bats and snakes, similar to rabies, and act as a reservoir for coronaviruses [7]. These zoonotic viruses infect a variety of wild 
animals and maintain the viral pool within the animal community. Based on genetic glycoprotein analyses, snakes, bats and civets have been identified as key hosts [8]. Following a possible recombination and transmission may have involved direct contact of flesh or consumption of raw or partially cooked meat from infected animal could transfer this virus to humans. Nevertheless, the origin of this virus seems unlikely from this source and it is far from clear.

\section{Brief History of this New Coronavirus}

The current outbreak of coronavirus (named, Wuhan coronavirus, 2019-nCoV, and later, COVID-19), originated from Wuhan city in Hubei Province, China [9]. The virus has spread to other provinces within China and to other countries primarily through international travel. Wuhan health Authority failed to close its boarder till in excess of four million people moved out of the province, including those who were carrying the virus. Travelers should be concerned of COVID-19 outbreak, because of the potential for travel-related exposure and contracting the virus and airport closures and flight cancellations affecting travel plans [10]. In December 2019, Chinese health authorities first reported to the World Health Organization (WHO), a cluster of persons with viralmediated pneumonia. When a certain gene mutation occurs within the viral genome, the virus could acquires the capacity to spread to humans and also between humans [10].

It is important that the WHO, Wuhan Health Department, and the United Stated Centres for Disease Control (US CDC) and China's Centre for Disease Control and Prevention (CCDC) to work in collaboration and openly share data and provide authentic updates and advice to the other countries [11], including sharing reliable information using social media platforms. The above is particularly important, as the majority of the mainstream news and social media continue to disseminate fabricated and unreliable information with reference to COVID-99. Many such media have exaggerated claims and predictions causing fear mongering. For example, an Australian outlet claimed that it the Wuhan coronavirus epidemic will lead to deaths exceeding 65 million in the absence of reliable data [12].

\section{Recent Evolution of COVID-19}

Coronavirus belongs to a group of viruses, generally associated with common cold [13]. Genetically, it is related to viruses causing severe acute respiratory syndrome (SARS) and the Middle East respiratory syndrome (MERS). The 2019-nCoV has typical genome structure of coronavirus. It belongs to the cluster of betacoronaviruses, including Bat-SARS-like (SL)-ZC45, Bat-SL ZXC21, SARS-CoV and MERS-CoV [13]. January 2020, the World Health Organization temporarily named the new virus as 2019 novel coronavirus (2019nCoV.2) [14], of which the gene sequencing data were reported in the GenBank on the same week [13] It is a long, single-stranded positive-sense RNA [+ssRNA) ( 30kb) with 5 '-cap structure and 3 '-poly-A tail; genome size of $\operatorname{CoV}(\sim 30 \mathrm{~kb})]$ that is described in, Coronaviruses: genome structure, replication, and pathogenesis [13]. COVID-19 virus predominately affecting the epithelial cells of the pulmonary and the gastrointestinal tract, and cardiovascular endothelial cells [10]. Cause of death in COVID-19 is multifactorial and include, cytokine storm, pulmonary micro-embolism, oedema and pneumonia (available on Virological.org) [15], but other organs, including the central nervous system can get affected. 2019$\mathrm{nCoV}$ is part of a family of coronaviruses that includes the common cold, severe acute respiratory syndrome (SARS), and Middle East respiratory syndrome (MERS) [14]. Since first identified in Saudi Arabia in 2012, and around 34\% of people reported as infected with MERS have died ( 858 of 2,494 cases; MERS R0 is less than one). The SARS outbreak led to 8,098 identified cases and 774 deaths (9.6\%; SARS had an R0 of 2-5). SARS disappeared as quickly as it appeared in 2002-03.

\section{Symptoms of COVID-19}

The symptoms generally consist of cough, mild fever, and shortness of breath. However, these symptoms are similar to those of the common cold and flu and thus may be difficult to use for diagnosis of the virus [10]. General signs and symptoms are fever, severe cough, shortness of breath, and pneumonia; these are no different from those of other respiratory viruses and bacterial infections. A smaller number of infected people present with non-respiratory symptoms, such as nausea, vomiting, or diarrhoea, similar to those associated with enteric viruses. Most affected people recover within ten days. A small minority of people, especially those with immune compromised status, those taking medications such as prednisolone, and infants and the elderly, could develop serious infection, such as infectious pneumonia, and may experience a secondary bacterial infection [14].

\section{Contagiousness of Coronavirus}

Coronavirus spread mostly through the air. These enveloped viruses carried in small droplets that are produced when a sick person breathes, talks, coughs, or sneezes. Because of the airborne nature, the common routes of acquiring this disease following the exposure to affected people in crowded and enclosed places, public transportations, and air travel. To acquire COVID-19, one needs to be in close contact with a person infected with the coronavirus. In the absence of such contact, one with casual contact or visiting a shopping centre is unlikely to contract the coronavirus. Coughs or sneezes from an infected person likely to spread higher viral loads and thus exceedingly infectious. COVID-19 spread occurring through droplets: while larger droplets travel up to 6 feet $(\sim 2$ meters) before scattering, whereas smaller droplets can float in the air and infect people in distance up to 30 feet.

Coronaviruses can also be present in the stools of affected individuals. However, transmission via the faecal-oral route root while possible, is less likely. Other viral diseases, such as measles and varicella (chickenpox), and bacterial diseases such as tuberculosis are organisms that can travel in much smaller droplets over greater distances through the air and are highly infectious. Whereas, viruses, such as HIV and hepatitis, are transmitted only through direct contact with the bodily fluids of an infected person. COVID-19 is considered moderately infectious and somewhat similar to severe acute respiratory syndrome (SARS), which became a local epidemic in China in 2003. SARS infected more than 9,000 
people, and 774 died of it. Infectiousness determines how easily and rapidly a virus is transmitted from person to person. In the absence of proper precautions, based on the contact information, it has been estimated that one person with the COVID-19 could infect up to six people through a single exposure event.

\section{Measures to Prevent Infection}

During the wintertime, as happened during 2020, people are much more likely to get respiratory viral illnesses, such as influenza $\mathrm{B}$ (the flu) than other viral diseases. Following the basic infectious disease prevention principles are recommended to curtail the spread of such viruses. Public health principles, such as wearing face masks in public, regular hand washing with soap and water or medical grade hand sanitizers and covering the nose and mouth with disposable tissues or your inner elbow when coughing or sneezing as essential basic steps for everyone to follow. Since the COVID-19 also can enter the body through mucous membranes, it is recommended not to touch eyes, nose, or mouth (mucosal surfaces) and genito-anal regions without first washing hands thoroughly with soap and water to avoid self-inoculation. In addition, staying at home when one has flu-like symptoms or an unexplained fever, and keeping distance from people who have signs of a respiratory tract infection, including runny nose, coughing, and sneezing is strongly recommended. Every person is susceptible to contract COVID-19. With the efficient public health measures implemented rapidly in many countries, the spread of COVID-19 might have been somewhat curtailed. Human-to-human transmission can be curtailed by efficient public health procedures, such as efficient tracking of individuals who are in contact with an infected person, isolating sick people (quarantine measures), and large-scale public education. In this regard, health authorities/departments in many countries are taking effective public health measures to curtail this disease that can have a major impact on the economy.

\section{Transmission of the Disease Via Air Travelers}

Initially, the virus spread from China to its other provinces and then to other countries via tourists, visitors, and transit workers and students returning home from visiting Wuhan and other infected regions. In countries, such as the United States, individuals arriving from areas where the coronavirus was the main reason for the spread of COVID-19 in the country. From such travellers, people can become infected even though they may not have visited places known to have the disease. Poorly managed quarantine centre is another source for the spready of COVID-19 to otherwise healthy people, which is highly unethical. Although a 14-day quarantine period is recommended by the WHO, there are instances people contracted the virus while in these centres and thus leaving these centres infectious. The number of people infected with COVID-19 in China has exponentially increased within weeks, even faster than that occurred with other SARS viruses. Nevertheless, while an average person with good innate immune system should not panic, there are valid reasons to cancel travel plans, person to person interactions of children and youth with the elderly and visiting gatherings should be curtailed to minimize the spread of the virus.

\section{Mortality Associated with the Coronavirus}

As per the WHO, currently the death rate associated with COVID-19 is approximately 3\%, which is tenfold less than that associated with the SARS virus. Whereas, the reported death rates from most countries are between 0.5 and $1.5 \%$. Countries with a larger proportion of older population and hypovitaminosis D have the highest COVID-19 related complications, ICU occupancy, and death rates. However, if the virus continues to mutate, it might acquire virulent characteristics. Mortality statistics from COVID-19 vary widely between countries, is positively corelated with the age, ethnic minority group with darker skin colour, and living far away from the equator. Because of too little PCR testing performed in developing countries, it is estimated that between 10 and 40 people in the community could be infected for each hospital-based PCR positive patient.

Of these COVID-19 infected people, the majority could be asymptomatic but carries the virus, thus infectious. The latter group usually have good innate immune system and so, develop antibodies again the virus and able rapidly overcome from the COVID-19 infection with relatively milder illness or even symptomatic. Because PCR testing is not performed in this group of people, the reported prevalence of COVID-19 is underestimated. It is also possible that deaths caused by COVID-19 are documented as from other diseases, such as bronchitis or pneumonia or even myocardial infarction and not attributed to COVID-19. While the contagiousness is higher, the mortality rate associated with COVID-19 is less than that associated with SARS or Middle East Respiratory Syndrome (MERS). SARS with a 5-day incubation period had a $10 \%$ mortality rate, for MERS it was 33\%, whereas, for COVID-19 overall death rate is approximately 1.5\%. Most people who died of a coronavirus had underlying chronic health problems, especially pulmonary can cardia and deranged immune systems and over $95 \%$ had hypovitaminosis D.

\section{Incubation Periods of Covid-19 Coronavirus and Other Recent Viral Outbreaks}

Diseases such as the common cold and influenza have a 2 or 3 day incubation period and signs and symptoms lasts for between 3 and 6 days. The disease is most contagious during the latter part of the incubation and early part of the symptomatic period. Although there are few signs and symptoms present during the critical incubation period, so as with the asymptomatic carriers of COVID-19, they are infectious to others during that period. It the case with COVID-19, the incubation period (prior to manifesting signs and symptoms) in part depends on the status of vitamin D and innate immunity and estimated to be between 3 and 14 days. Because of the lack of clear-cut manifestations, in contrast to highly infectious diseases such as measles and chickenpox, infectivity of coronaviruses can extend throughout the entire incubation period. 
This increased the likelihood of an infected person could spread the disease to others; RO more than 1 [16].

\section{Developing Vaccines and Antiviral Agents Against COVID-19}

In general, the development of an effective vaccine for a disease affecting humans takes at least 1 year. By the time a good vaccine is developed, the current COVID-19 outbreaks are likely to be over. For the SARS outbreak (2003), it took 20 months to develop a vaccine that was ready for human clinical trials. However, by that time, the disease was well under control and the vaccine was not even effective [16]. Whereas in 2015, in contrast, it took only 7 months to develop a vaccine for the Zika virus outbreak. The genome of COVID-19 has been sequenced and candidate proteins have been identified in the early stages of the pandemic $[17,18]$. Despite this and having the advantages of new technology, a vaccine that is approved for human clinical trial is only emerging now, after 6 months. It is not clear whether any of the vaccines under development would be safe, affordable to most people, and effective against COVID-19.

Preliminary data using animal models suggest that broadspectrum antivirals, such as an RNA polymerase inhibitor, remdesivir as well as lopinavir/ritonavir and interferon might be effective against 2019-nCoV and previously reported for MERSCoV. Despite the hypes and propaganda, recent studies reported the lack efficacy of these anti-viral agents. Therefore, researchers are experimenting the use of nucleic acid vaccine platform approaches that previously used for generating vaccines against SARS-CoV or MERS-CoV. There are no approved or effective antiviral medication yet except for hydroxychloroquine that shows some potential. Therefore, the current treatment is supportive therapy and keeping affected persons comfortable and alive for a natural recovery through own immune system $[16,19]$. A smaller number of people with high viral loads and/or inefficient or compromised immune systems likely to need management in intensive care unit (ICUs).

\section{What Can Be Done Right Now to Reduce Covid-19 Risk} and Severity?

COVID-19 must be diagnosed at earliest possible to minimize complications and to prevent infecting others. This would allow effective isolating PCR positive patients, real-time surveillance, providing supportive treatments and dynamic guidance to avoid unnecessary panic to the families and the community [10]. For individuals, good personal hygiene, proper use of fitted masks, avoiding crowded places, and providing safe ventilation in rooms and buildings will reduce risks of COVID-19 infection. Adequate serum 25(OH)D concentrations are associated with reduced incidence and the severity of many enveloped viruses [20], such as herpes zoster, Epstein-Barr, hepatitis, Ebola, HIV, dengue, measles, and mumps [21,22]. Multiple studies have reported administration of an oral dose of 50,000 IU of vitamin D reduces the risks of having influenza and COVID-19 [23,24]. Vitamin D adequacy also reduces severity of coronaviral infection associated pneumonia. Whereas the risks of administering vitamin D is minuscule [25-27].

Vitamin D is known to improve the immune system; its deficiency increases the vulnerability to viral infections, such as colds [21,22]. In most people, serum 25(OH)D concentrations are lowest during winter months, unless supplements are taken. Levels begin to rise at early summer. Considering its many biological and physiological aspects, vitamin D and its multiple immunoregulatory actions $[20,21]$, it is not surprised the significant effects of vitamin D in controlling COVID-19 and other respiratory viruses. To have these benefits, is advisable to maintain serum $25(\mathrm{OH}) \mathrm{D}$ concentrations in excess of $30 \mathrm{ng} / \mathrm{mL}(75 \mathrm{nmol} / \mathrm{L})$, preferably over $40 \mathrm{ng} / \mathrm{mL}$.

\section{Conclusion}

Prevention and minimizing the complications associated with the virus are the best option during the current COVID-19 pandemic. Proper functioning immune system is essential to overcome COVID-19, which is facilities by having vitamin $\mathrm{D}$ adequacy. Considering the inability to diagnosis during its incubation period, people feeling unhealthy or having a fever might benefit from taking a loading dose of vitamin D of between 100,000 (two 50,000 IU capsules) and 400,000 international units (IU) either as a single dose or daily doses of one 50,000 $(60,000)$ IU capsule for a week, or taking one capsule, once a week for eight weeks, which should be followed with a suitable maintenance dose, such as between 2,000 and 5,000 a day. This would allow strengthening the immune system and protect other body systems within two to four days of administration. Mentioned approaches reduce the incidence, severity of the illness, deaths, and expedite the recovery. A single high dose or a higher dose taken a week apart as described can be administered in hospitals or at community settings and can repeat after four months. It is highly unlikely to develop any adverse effects by using the mentioned doses of vitamin D. Toxicity will not develop unless taking doses higher than 20,000 IU daily, for several months or taking 1 million IU for a few days as have happened due to mistaken use. In addition to been highly cost-effective, its benefits Potential benefits far outweigh any minute risk to an individual.

\section{References}

1. Diaz JH (2020) Hypothesis: Angiotensin-converting enzyme inhibitors and angiotensin receptor blockers may increase the risk of severe COVID-19. Journal of Travel Medicine 27(3): 41.

2. Li J, Jie G, Ya PX, Tong LZ, Yao YJ, et al. (2007) Expression of severe acute respiratory syndrome coronavirus receptors, ACE2 and CD209L in different organ derived microvascular endothelial cells. Zhonghua Yi Xue Za Zhi 87(12): 833-837.

3. Chu PL, Thu HL (2014) Role of collectrin, an ACE2 homologue, in blood pressure homeostasis. Curr Hypertens Rep 16(11): 490.

4. Patel SK, Elena V, Melanie F, Bryan W, Terase FL, et al. (2014) From gene to protein-experimental and clinical studies of ACE2 in blood pressure control and arterial hypertension. Front Physiol 5: 227.

5. Menachery VD, Boyd LY, Kari D, Sudhakar A, Lisa EG, et al. (2015) A SARS-like cluster of circulating bat coronaviruses shows potential for human emergence. Nature Medicine 21(12): 1508-1513. 
6. Hoffmann M, Hannah KW, Simon S, Nadine K, Tanja H, et al. (2020) SARSCoV-2 cell entry depends on ACE2 and TMPRSS2 and is blocked by a clinically proven protease inhibitor. Cell 181(2): 271-280.

7. Zhou P, Xing LY, Zheng LS (2020) A pneumonia outbreak associated with a new coronavirus of probable bat origin. Nature 579: 270-273.

8. Wei J, Wei W, Xiaofang Z, Junjie Z, Xingguang L, et al. (2020) Crossspecies transmission of the newly identified coronavirus 2019-nCoV. J Med Virol 92(4): 433-440.

9. WHO (2019) Novel coronavirus (2019-nCoV).

10. Wimalawansa SJ (2020) Global epidemic of coronavirus-COVID-19: What can we do to minimize risks? European J Biomed \& Pharma Sci $7(3): 432-438$.

11. Gralinski LE, Menachery VD (2020) Return of the Coronavirus: 2019nCoV. Viruses 12(2): 135.

12. BI A (2020) A viral pandemic could kill 65 million people.

13. Chen Y, Q Liu, D Guo (2020) Emerging coronaviruses: Genome structure, replication, and pathogenesis. J Med Virol 92(4): 418-423.

14. Paules CI, Marston HD, Fauci AS (2020) Coronavirus infections-more than just the common cold. JAMA 323(8): 707-708.

15. Holland M, Negrón, Mitchell D, Ivancich S, Jennings M, et al. (2020) Preliminary in silico assessment of the specificity of published molecular assays and design of new assays using the available whole genome sequences of 2019-nCoV.

16. Wimalawansa SJ (2020) What modelling and reproduction numbers are useful in predicting COVID-19 spread? Can J Biomed Res \& Tech 3(3): $1-4$

17. Behrmann 0, Spiegel M (2020) COVID-19: From rapid genome sequencing to fast decisions. Lancet Infect Dis S1473-3099(20): 3058030586.

18. Licastro D, Sreejith R, Simeone DM, Ludovica S, Pierlanfranco DA, et al. (2020) Isolation and full-length genome characterization of SARS-CoV-2 from COVID-19 cases in Northern Italy. J Virol 94(11).
19. Wimalawansa SJ (2020) Fighting against COVID-19: Boosting the immunity with micronutrients, stress reduction, physical activity, and vitamin D. Nutrition and Food Science Journal (Sci Literature) 3(1) (126): 1-4.

20. Beard JA, Bearden A, Striker R (2011) Vitamin D and the anti-viral state. J Clin Virol 50(3): 194-200.

21. Beard JA, Bearden A, Striker R (2011) Vitamin D and the anti-viral state. J Clin Virol: The official publication of the Pan American Society for Clinical Virology 50(3): 194-200.

22. Gunville CF, Mourani PM, Ginde AA (2013) The role of vitamin D in prevention and treatment of infection. Inflamm Allergy Drug Targets 12(4): 239-245.

23. Kow CS, Hadi MA, Hasan SS (2020) Vitamin D supplementation in influenza and COVID-19 infections comment on: "Evidence that vitamin D supplementation could reduce risk of influenza and COVID-19 infections and deaths" Nutrients 2020, 12(4), 988. Nutrients 12(6): 1626.

24. Grant WB (2010) Vitamin-D supplementation could reduce the risk of type a influenza infection and subsequent pneumonia. Pediatr Infect Dis J 29(10): 987.

25. Boucher BJ (2020) Adjustments in analyses of vitamin D status, allowing for vitamin D determinants, for Covid-19 risks. Diabetes Metab Res Rev p. e3375.

26. Miliku K, Anna V, Laura MB, John JMcG, Darryl WE, et al. (2016) Maternal vitamin D concentrations during pregnancy, fetal growth patterns, and risks of adverse birth outcomes. Am J Clin Nutr 103(6): 1514-1522.

27. Goldsmith JR (2015) Vitamin D as an immunomodulator: Risks with deficiencies and benefits of supplementation. Healthcare (Basel) 3(2): 219-232. 